(RESEARCH ARTICLE)

\title{
Fabrication and characterization of superhydrophobic coatings on cotton fabrics using silica nanoparticles for self-cleaning applications
}

\author{
Kanwal Zakir 1, Syeda Saniya Maham Shahzadi 1, Shafqat Rasool 1, Zakia Kanwal 2, Saira Riaz 1, Shahzad \\ Naseem ${ }^{1}$ and Muhammad Akram Raza ${ }^{1, *}$ \\ ${ }^{1}$ Centre of Excellence in Solid State Physics, University of the Punjab, Quid-e-Azam Campus, Lahore 54590, Pakistan. \\ 2 Department of Zoology, Lahore College for Women University, Jail Road, Lahore 54000, Pakistan.
}

Publication history: Received on 18 November 2020; revised on 30 November 2020; accepted on 03 December 2020

Article DOI: https://doi.org/10.30574/wjarr.2020.8.3.0458

\begin{abstract}
The marvelous self-cleaning properties of lotus leaves and their superhydrophobic nature inspire the researchers to mimic such functionalities on cotton fabrics. The present work aimed to prepare and characterize true non-sticky superhydrophobic properties on cotton samples. The required combination of micro and nanostructure roughness was achieved by depositing the silica nanoparticles (SiNPs) on cotton surfaces by dip-coating method. To enhance adhesion and durability of silica particles with cotton, SiNPs were functionalized with 3-Aminopropyltriethoxysilane (APTES) before deposition. The surface energy of SiNPs coated cotton samples was lowered by functionalizing with $1 \mathrm{H}, 1 \mathrm{H}, 2 \mathrm{H}$, 2H-Perfluorooctyltriethoxysilane (PFOTS). The morphology, surface chemistry and wettability characterizations of prepared samples were performed by scanning electron microscope (SEM), energy dispersive X-ray analysis (EDX) and contact angle measurement system (goniometer) respectively. SEM micrographs revealed the nice decoration of SiNPs (having particles size range $90-150 \mathrm{~nm}$ ) on the cotton fibers leaving to the hierarchical roughness. The EDX analysis confirmed the coatings of SiNPs and PFOTS. Contact angle measurements exhibited the superhydrophobic nature of prepared surfaces by static water contact angle of $157 \pm 2^{\circ}$ and sliding angle of less than $5^{\circ}$. The functionalized SiNPs/PFOTS cotton fabrics can effectively be used for self-cleaning applications.
\end{abstract}

Keywords: Superhydrophobicity, Contact angle, Cotton fabrics, Silica nanoparticles, Self-cleaning

\section{Introduction}

Superhydrophobic coatings have achieved great attraction owing to their potential applications in non-wetting, antibacterial, anti-icing and self-cleaning [1-4]. Superhydrophobicity can be defined as a non- wetting state having water contact (CA) greater than $150^{\circ}$ and it can further be divided into two categories as: 1) sticky superhydrophobic surfaces which have $C A>150^{\circ}$ and sliding angle $(S A)$ less than $\left.5^{\circ}, 2\right)$ non-sticky superhydrophobic surfaces which have $C A>150^{\circ}$ and $S A<5^{\circ}[1]$. Both sticky and non-sticky superhydrophobic surfaces have their importance in practical applications.

Controlling the surface wettability depends strongly upon two major factors; surface energy and surface roughness. For achievement of superhydrophobic behavior of the surface a suitable combination of both factors is very important. Furthermore, the role of hierarchical (a combination of micro- and nano- level) roughness cannot be ignored as revealed by microstructural examination of naturally occurring superhydrophobic surfaces such as lotus leaf, rice leaf and butterfly wings [1,5]. In the case cotton fabric surfaces, micro-level roughness already exists due to the fibrous structure of woven fabrics. Researchers only need to add secondary nano-level roughness to achieve the hierarchical structures. For this purpose, fabrication of different types of nanostructures on the cotton fibers is carried out; for example deposition of spherical shaped metallic (silver, gold) nanoparticles [6,7], coatings of ZnO nanorods [8], incorporation of carbon nanotubes [9], combination of $\mathrm{TiO}_{2}$ nanostructures [10] and decoration of silica nanoparticles [2, 3, 11]. But the use of SiNPs can be preferred because surface chemistry of fibers of cotton and silica matches very well and both have

\footnotetext{
* Corresponding author: Muhammad Akram Raza akramraza.cssp@pu.edu.pk

Centre of Excellence in Solid State Physics, University of the Punjab, Quid-e-Azam Campus, Lahore 54590, Pakistan.
} 
OH-group which helps in applying the same material for lowering the surface energy instead of using different lower energy compounds for this purpose $[12,13]$. Various approaches are used to prepare superhydrophobic coating on the cloth such as dip-coating methods, Chemical bath deposition, Wet chemical etching, Electrophoretic deposition, Electro spinning, spray coating, chemical vapor deposition and plasma etching [14]. Similarly to deposit the SiNPs on cotton fabrics different methods are in practice such as electrostatic layer-by-layer (LbL) assembly [2], padding [15] and dipcoating [16].

In present work, we used the wet chemistry approach 'sample immersion method' which is a slightly modified form of the dip-coating technique to deposit the silica nanoparticles on the cotton fabrics. Our method provides various advantages for example, large samples can be treated with SiNPs, additional binding agent can be added in the same step and even the temperature of the solution can also be controlled during the deposition process. Finally the hydrophobization of silicate cotton sample was also done by treating with fluoroalkylsilane. The combination of surface morphology and surface energy allowed tuning the surface wettability of cotton samples from superhydrophilic to nearsuperhydrophobic to non-sticky superhydrophobic nature.

\section{Material and methods}

\subsection{Material and chemicals}

The pristine, plain-weave cotton fabrics used on this work was obtained from local textile mills. Tetraethylorthosilicate (TOES), 3-Aminopropyltriethoxysilane (APTES), Ammonium hydroxide ( $\mathrm{NH}_{4} \mathrm{OH}$ ), Sodium Hydroxide (NaOH), Ethanol, $1 \mathrm{H}, 1 \mathrm{H}, 2 \mathrm{H}, 2 \mathrm{H}$-Perfluorooctyltriethoxysilane (PFOTS), and Deionized (DI) water. All the chemicals used were of research grade and used without any further purification.

\subsection{Synthesis of silica nanoparticles}

Silica nanoparticles (SiNPs) used for the decoration of cotton fibers were prepared by suing the well-known Stöber method mentioned elsewhere [17] with some modifications. First of all, $50 \mathrm{ml}$ of ethanol and $20 \mathrm{ml}$ of aqua ammonia were mixed in a $500 \mathrm{ml}$ flask under magnetic stirring (250 rpm) and heated to $40{ }^{\circ} \mathrm{C}$. Then $4 \mathrm{ml}$ TOES was added dropwise (one per second) with the help of dropper. The solution was further heated under constant stirring for one hour. The color of the solution changed from transparent to white turbid. The silica particles from this white suspension were obtained by centrifugation at $6000 \mathrm{rpm}$ for $20 \mathrm{~min}$ (Figure 1). The obtained silica particles were washed thrice with ethanol-water and finally stored for further use. Different stages during the preparation of SiNPs are shown in Figure 1.
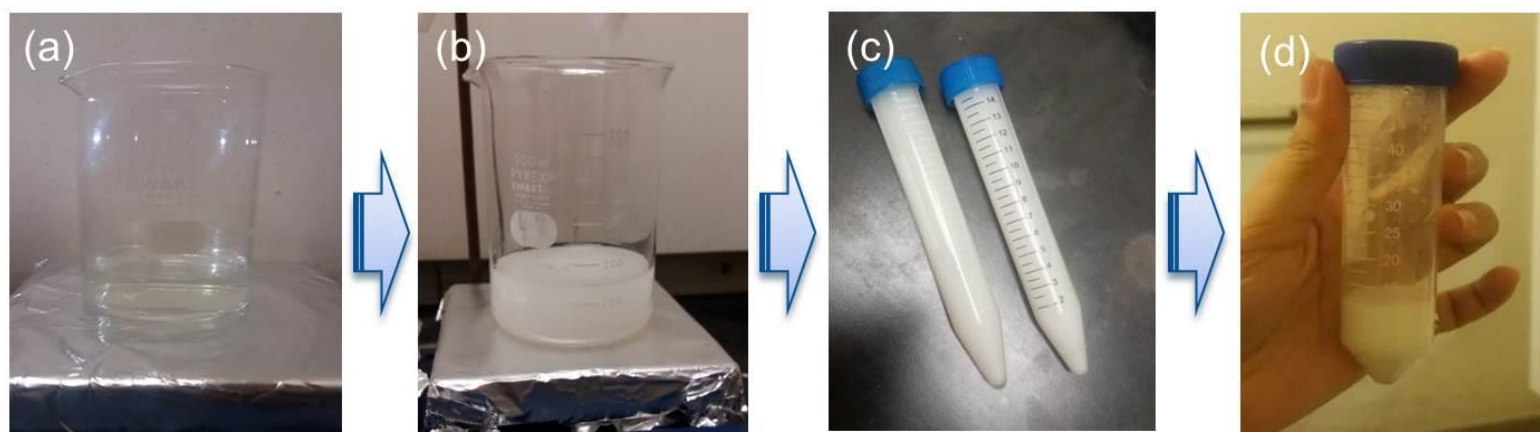

Figure 1 Different stages during the preparation of silica nanoparticles (SiNPs)

\subsection{Deposition of silica nanoparticles on cotton fabrics}

First, pristine cotton fabrics was washed twice with non-ionic detergent and bleached to clean it properly then dried at room temperature and finally cut into $3 \mathrm{~cm} \mathrm{x} 4 \mathrm{~cm}$ samples. Before depositing the SiNPs on cotton, SiNPs were functionalized with APTES to enhance the adhesion of SiNPs with cotton fibers. For this purpose, a literature reported method [18] with some alterations was used for the APTES treatment. Briefly, first $2 \% \mathrm{v} / \mathrm{v}$ solution of APTES in ethanol was prepared and the SiNPs were added and mixed well by stirring, the solution, color changed into milky shade. Then clean cotton samples were immersed into this milky suspension overnight and covered with aluminum foil. Next day, all cotton samples were taken out from the suspension and dried in oven at $80^{\circ} \mathrm{C}$ for $10 \mathrm{~min}$. In the next step, all samples were padded at $110^{\circ} \mathrm{C}$ under the padder pressure of $2 \mathrm{~kg} / \mathrm{cm}^{2}$. Finally samples were cured at $120^{\circ} \mathrm{C}$ in oven for $40 \mathrm{~min}$. 

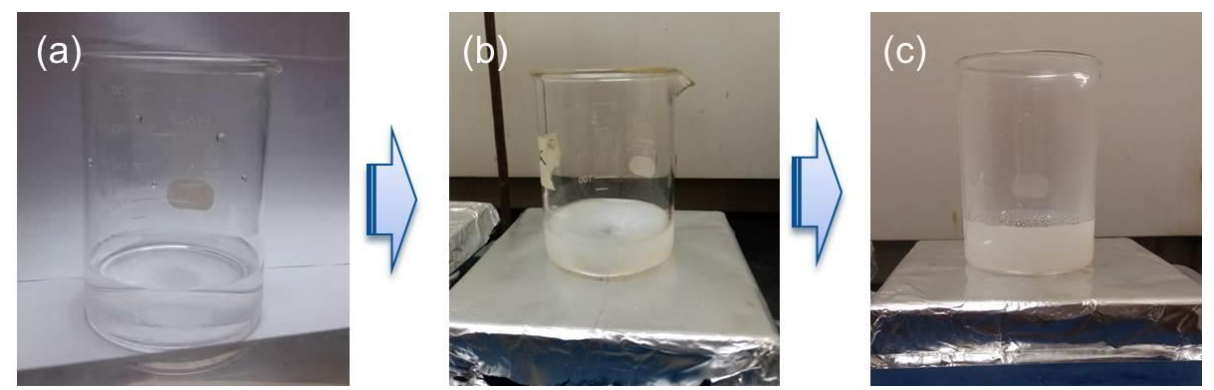

Figure 2 Different stages during APTES treatment of silica nanoparticles

\subsection{Hydrophobization of Cotton samples with Fluoroalkylsilane (PFOTS)}

In order to further lower the surface energy, SiNPs coated cotton samples were treated with highly fluorinated solution of PFOTS using our already reported methods [19]. Briefly, 3\% v/v solution of PFOTS was prepared in ethanol and all samples were immersed into it. The sample remained dipped in PFOTS solution for $3 \mathrm{hrs}$ under a constant stirring of $70 \mathrm{rpm}$. Finally all samples were taken out from the solution, dried and cured in finance box at $150^{\circ} \mathrm{C}$ in air for $10 \mathrm{~min}$.

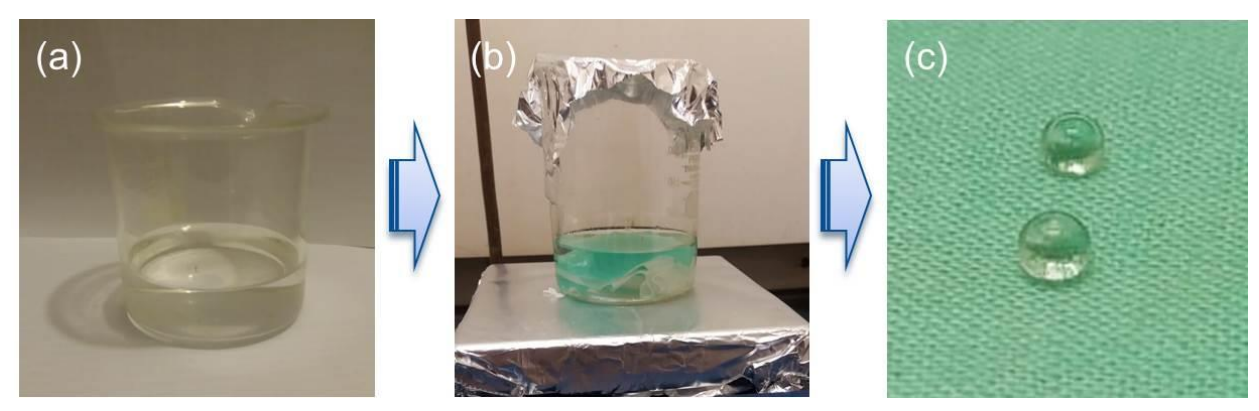

Figure 3 Different stages during PFOTS treatment of SiNPs coated cotton samples

A summary of all the steps involved in the fabrication of superhydropbonic coatings on the cotton sample is schematically presented in figure 4 .

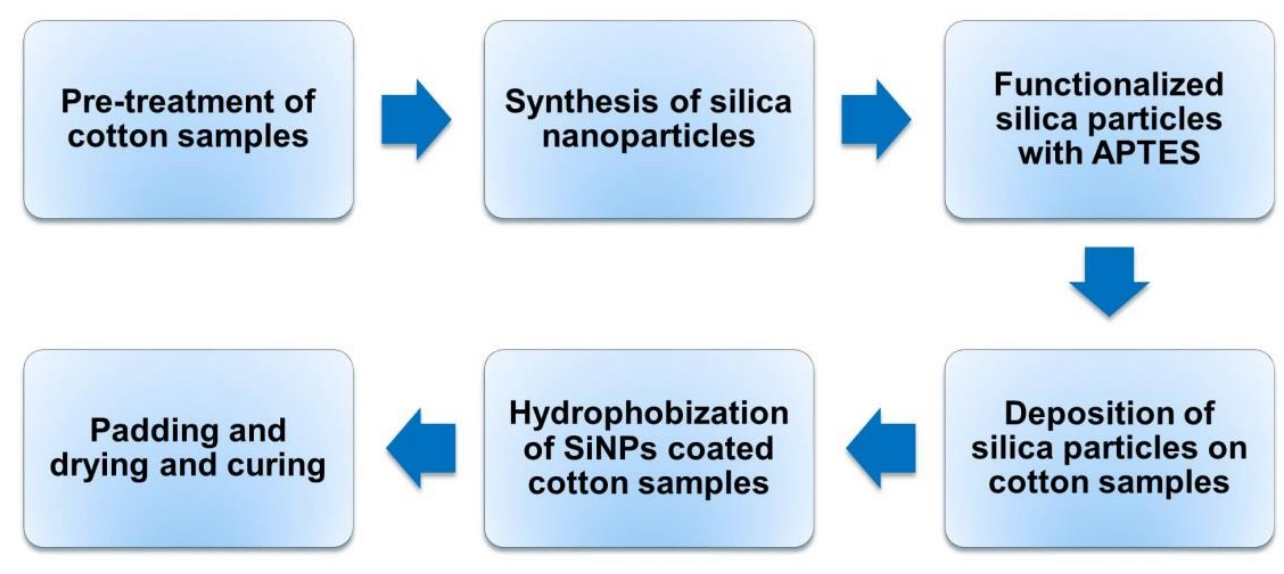

Figure 4 schematically shown different stages during the preparation of superhydropbonic cotton samples

\subsection{Characterizations of functionalized cotton samples}

In order to study the morphology of pristine, SiNPs coated and PFOTS treated cotton samples Scanning Electron Microscope (SEM, Carl ZEISS EVO 10, Germany) was used. Energy dispersive X-ray analysis (EDX) analysis of the cotton samples at each stage during the treatment was also conducted to study the changes occurring in the surface chemistry. 
The surface wettability was also examined at each stage by measuring the contact angle using the Contact Angle Measurement System (SEO-Phoenix-MT(A), Korea).

\section{Results and discussion}

Controlling surface wettability is an important parameter for designing the functional surfaces for practical applications. The comprehensive analysis of naturally occurring surfaces having wettability from superhydrophilic $\left(\mathrm{CA}<10^{\circ}\right)$ to superhydrophobic $\left(\mathrm{CA}>150^{\circ}\right)$ nature revealed that surface roughness especially hierarchical structures plays vital role than surface chemistry in controlling the wetting properties $[20,21]$.

Thus in our work, we coated SiNPs on the cotton fibers to achieve the required multi-leveled roughness and PFOTS treatment was carried to minimize the surface energy which lead to the non-wetting state of cotton surfaces. Surface structures of cotton samples before and after different treatments was examined by Scanning electron microscopy (SEM) and the wetting properties at each stage was studied using the CA measurements. The obtained SEM micrographs and CA results are discussed in the light of existing literature.

The morphology of pristine cotton sample, as shown in Figure 5(a,b), was found of typical smooth longitudinal fibril structure. Most of the fibers were free from any contamination except very few particles which could be some dust or some other impurities as can be noticed in the magnified view (Figure 5b). The pristine cotton samples, exhibited complete wetting (superhydrophilic) with $\mathrm{CA}<10^{\circ}$ (Figure $4 \mathrm{c}$ ). As the cotton samples were made of cellulose fibers which own a large number of hydroxyl $(\mathrm{OH})$ groups and thus water readily absorbed and spread on untreated cotton due to hydrogen bonds with water molecules [22. 23]. This wicking of water caused the complete wetting of pristine cotton samples as shown in figure 5c.
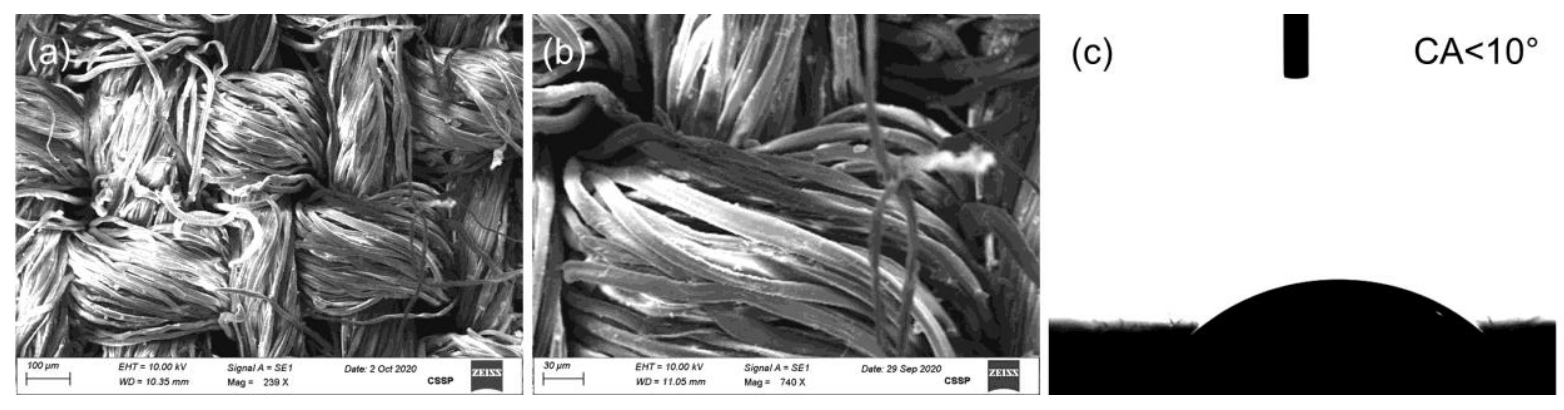

Figure 5 (a) SEM image of pristine cotton sample revealing the longitudinal fibril structure, (b) magnified SEM view showing the smooth and clean fibers. (c) Image of water CA on untreated cotton samples indicating the superhydrophilic behavior.

The deposition of APTES-functionalized SiNPs on cotton samples can be observed in Figure 6. A nice coverage of SiNPSs on the whole fibrous structure indicates almost uniform coating of particles ((Figure 6a). The size of the synthesized SiNPs was found in the range of 90-150 $\mathrm{nm}$ and shapes were almost spherical as can be noticed in the magnified SEM image (Figure 6b). However, at some places bunches of SiNPs can also be seen which could be due to agglomeration of nanoparticles creating the hierarchy of micro and nano-sized roughness. Furthermore, some places on the fibers can also be discerned without SiNPs indicating the hill-and-valley structure. Thus the decoration of SiNPs on the cotton fibers fashioned the hierarchical structures.

The surface wettability of pristine cotton samples after APTES-functionalized SiNPs coatings changed from superhydrophilic nature $\left(\mathrm{CA}<10^{\circ}\right)$ to near-superhydrophobic $\left(\mathrm{CA} \approx 147^{\circ}\right)$ as shown in Figure $6 \mathrm{c}$. The reasons of this increased CA can be explained as that the APTES modified the surface energy of both the cotton fibers and SiNPs. Because the APTES molecules can make hydrogen bonding from both ends with the OH-groups of fibers as well as silica NPs $[24,25]$. APTES is a lower energy chemical as compared to OH-groups and thus can reduce the energy of silica and cotton fibers by self-assembling of $\mathrm{NH}_{2}$-end groups. Therefore, a combination of enhanced roughness due to hydrophobized SiNPs and cotton fibers lead to the lowered surface wettability and increased the value of static water CA (Figure 6c). 

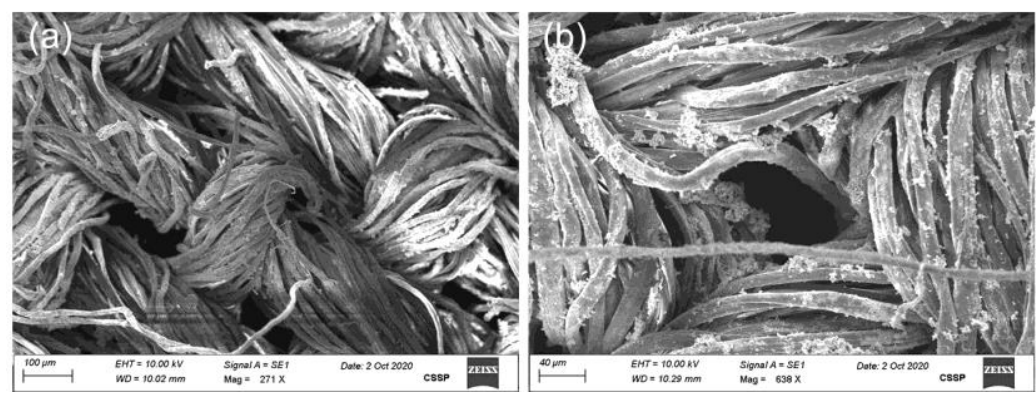

(c)

$\mathrm{CA}=146 \pm 2^{\circ}$

Figure 6 (a) SEM micrograph of cotton sample after APTES-functionalized SiNPs deposition, (b) magnified view revealing the nice coverage of SiNPs on cotton fibers. (c) Water droplet image on SiNPs coated cotton samples indicating the near-superhydrophobicity.

The morphology of the cotton samples after APTES-functionalized SiNPs coatings and PFOTS treatment is presented in Figure 7. The density of deposited NPs seemed to be enhanced. This could be the result of the polymerization due to the hydrolysis and condensation of saline groups during PFOTS treatment $[13,26]$. The size of SiNPs clusters also appeared to increase owing to this polymerization. Moreover, a close observation in the magnified view (Figure 7b) revealed a thick organic layer of saline groups which not only is hydrophobized by lowering the surface energy but also provided the structure stability due to polymerization. The value of static water CA further increased to $157 \pm 1^{\circ}$ exhibits the true superhydrophobicity of surfaces as shown in Figure 7c. Furthermore, the value of dynamic water droplet was found to be less than $5^{\circ}$ (Figure $7 \mathrm{~d}$ ) indicating the true non-sticky nature of cotton surfaces which is believed very important for self-cleaning applications.
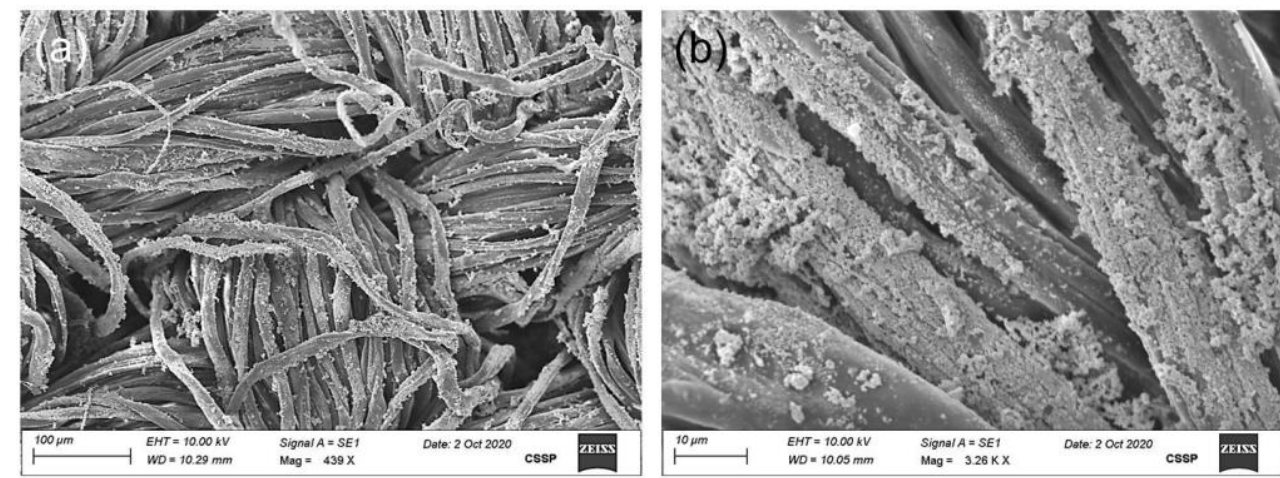

(c)

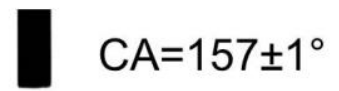

(d)
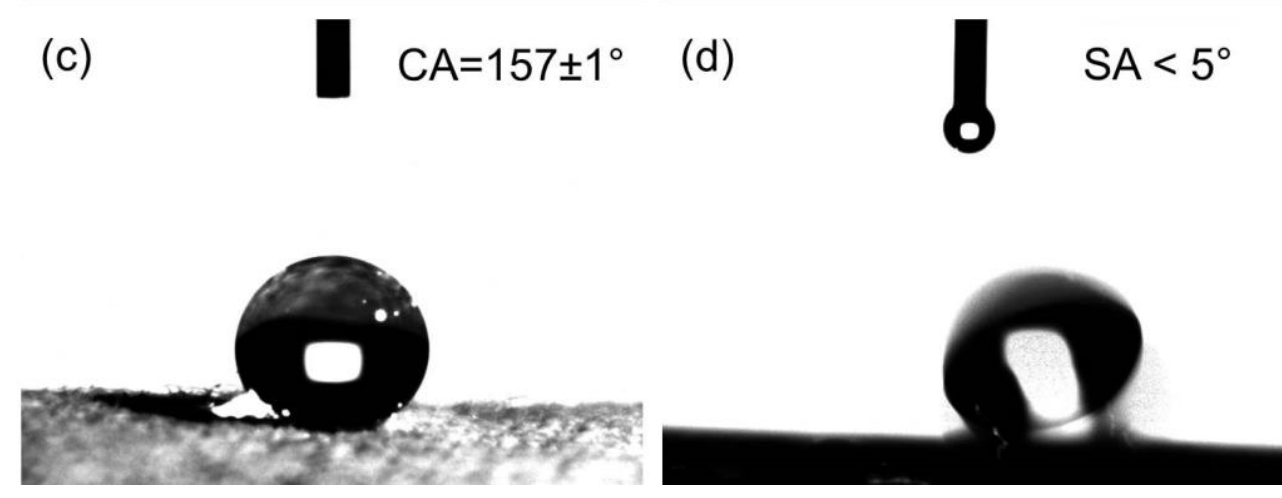

Figure 7 (a) SEM image showing the morphology of cotton sample after SiNPs deposition and PFOTS treatment, (b) magnified view showing full no loss of SiNPs on cotton fibers, (c) water droplet on SiNPs coated cotton sample after PFOTS treatment showing superhydrophobic behavior, (d) water droplet rolling off the prepared superhydrophobic cotton sample indicting non-sticky nature

As mentioned above, surface wettability can be controlled by tuning the surface roughness and surface energy. The treatment PFOTS lowered the energy of already developed hierarchical roughness owing to the fluorine end group. This ideal combination minimized the surface wettability and thus true superhydrophobicity achieved. Actually in hierarchical structures water cannot penetrate into the surface structures due the presence of air pockets and water 
droplet only touches the minimum places of the solid as explained by Cassie and Baxter in their model [27]. Since water droplet touches only very few places of solid structures and remains in 'floating state' on the air pockets and thus can easily roll off at a negligible angle [28]. That's why the value of SA on our prepared superhydrophobic cotton was less than $5^{\circ}$ (Figure $7 d$ ). A nice view of water droplets on the functionalized superhydrophobic cotton fabrics is shown in Figure 8, where droplets seem to sit on air pockets in 'floating' Cassie-Boxter state.

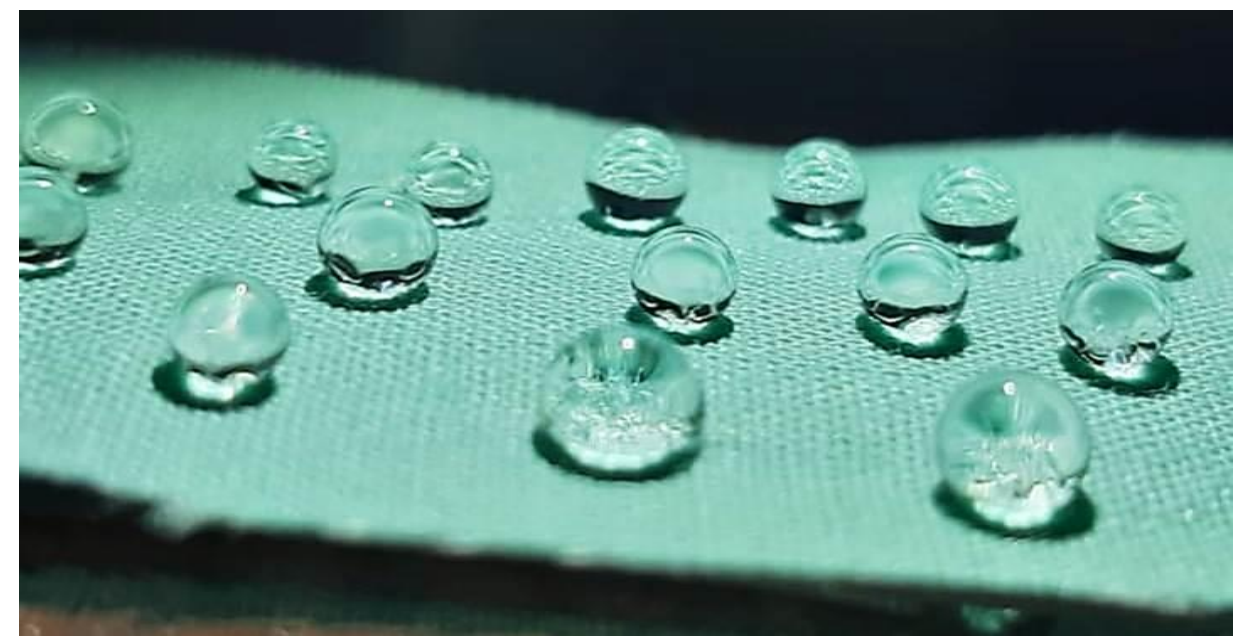

Figure 8 CCD image of different water droplet on the SiNPs coated cotton sample after PFOTS, showing the beautiful superhydrophobic view.

\section{Conclusion}

In this study, non-sticky superhydrophobic coatings were fabricated on pristine cotton fabrics using a wet chemical sample immersion method. The required hierarchical roughness was achieved by decorating the cotton fibers with APTES- functionalized silica nanoparticles. The surface energy of the fashioned structures was lowered by applying the highly fluorinated coatings of Perfluorooctyltriethoxysilane (PFOTS). The SEM morphological analysis revealed that the size of deposited SiNPs was in the range of 90-150 nm. The surface wettability of prepared cotton samples was assessed by measuring by static and dynamic water contact angle at different stages. The surface wettability of cotton samples was turned from superhydrophilicity $\left(\mathrm{CA}<10^{\circ}\right)$ to true superhydrophobicity $\left(\mathrm{CA}>150^{\circ}\right)$ with non-sticky behavior $\left(\mathrm{SA}<5^{\circ}\right)$. It was observed that a suitable combination of hierarchical structures and lower surface energy can provide true superhydrophobic behavior.

\section{Compliance with ethical standards}

\section{Acknowledgements}

The authors gratefully thank and acknowledge the financial support of Higher Education Commission (HEC) of Pakistan. The funding for this research was provided under the Project of National Research Program for Universities (Project no. HEC-NRPU-8019).

\section{Conflict of interest statement}

The authors declare no conflict of interest.

\section{References}

[1] Raza MA, Kooij ES, van Silfhout A, Zandvliet HJW, Poelsema B. A colloidal route to fabricate hierarchical sticky and non-sticky substrates. J. Colloid Interface Sci. 2012; 385(1);73-80.

[2] Zhao Y, Tang Y, Wang X, Tong Lin T. Superhydrophobic cotton fabric fabricated by electrostatic assembly of silica nanoparticles and its remarkable buoyancy. Appl Surf Sci. 2010; 256(22):6736-6742. 
[3] Sriramulu D, Reed EL, Annamalai M, Venkatesan TV, Valiyaveettil S. Synthesis and Characterization of Superhydrophobic, Self-cleaning NIR-reflective Silica Nanoparticles. Sci Rep.2016; 6:35993.

[4] Nosonovsky M, Bhushan B. Superhydrophobic surfaces and emerging applications: Non-adhesion, energy, green engineering. Curr Opin Colloid Interface Sci. 2009; 14(4):270-280.

[5] Koch K, Bhushan B, Jung YC, Barthlott W. Fabrication of artificial Lotus leaves and significance of hierarchical structure for superhydrophobicity and low adhesion. Soft Matter. 2009; 5(7);1386-1393.

[6] Wang T, Hu XG, Dong SJ.A general route to transform normal hydrophilic clothes into superhydrophobic. Chem. Commun. 2007; 46(18); 1849-1851.

[7] Gao YN, Wang Y, Yue TN, Weng YX, Wang W. Multifunctional cotton non-woven fabrics coated with silver nanoparticles and polymers for antibacterial, superhydrophobic and high performance microwave shielding. J. Colloid Interface Sci. 2021; 581:112-123.

[8] Xu BZ. Cai Z, "Fabrication of a Superhydrophobic ZnO Nanorod Array Film on Cotton Fabrics via a Wet Chemical Route and Hydrophobic Modification," Appl Surf Sci. 2008; 254(18): 5899-5904

[9] Hsieh CT, Chen WY, Wu FL. Fabrication and superhydrophobicity of fluorinated carbon fabrics with micro/nanoscaled two-tier roughness. Carbon. 2008; 46 (9): 1218-1224.

[10] Abbas M, Iftikhar $\mathrm{H}$, Malik MH, Nazir A. Surface Coatings of $\mathrm{TiO}_{2}$ Nanoparticlesonto the Designed Fabrics for Enhanced Self-Cleaning Properties. Coatings. 2018: 8(1):35.

[11] Jeong SA, Kang TJ. Superhydrophobic and transparent surfaces on cotton fabrics coated with silica nanoparticles for hierarchical roughness. Text Res J. 2016; 87(5):552-560.

[12] Raza MA, Kooij ES, van Silfhout A, Poelsema B. Superhydrophobic Surfaces by Anomalous Fluoroalkylsilane SelfAssembly on Silica Nanosphere Arrays. Langmuir 2010; 26(15);12962-12972.

[13] Raza MA, Zandvliet HJW, Poelsema B, Kooij ES. Hydrophobic surfaces with tunable dynamic wetting properties via colloidal assembly of silica microspheres and gold nanoparticles. J Sol-Gel Sci Technol. 2015; 74:357-367.

[14] Liu H, Gao SW, Cai JS, He CL, Mao JJ, Zhu TX, Chen Z, Huang JY, Meng K, Zhang KQ, Salem S. Al-Deyab SS, and Lai YK. Recent Progress in Fabrication and Applications of Superhydrophobic Coating on Cellulose-Based Substrates. Materials. 2016; 9:124.

[15] Daoud WA, Xin JH, Tao XM. Superhydrophobic Silica Nanocomposite Coating by a Low-Temperature Process. J Am Ceram Soc. 2004; 87(9):1782-1784.

[16] Li ZX, Xing YJ, Dai JJ. Superhydrophobic Surfaces Prepared from Water Glass and Non-Fluorinated Alkylsilane on Cotton Substrates. Appl Surf Sci. 2008; 254(7):2131-2135

[17] Stöber W, Fink A,Bohn E, Controlled growth of monodisperse silica spheres in the micron size range. J. Colloid Interface Sci. 1968; 26:62.

[18] Villa S, Riani P, Locardi F, Canepa F. Functionalization of $\mathrm{Fe}_{3} \mathrm{O}_{4}$ NPs by Silanization: Use of Amine (APTES) and Thiol (MPTMS) Silanes and Their Physical Characterization. Materials. 2016;9: 826.

[19] Raza MA, Kooij ES, van Silfhout A, Poelsema B. A Facile Colloidal Route for Superhydrophobic Films with Hierarchical Roughness. Progr Colloid Polym Sci. 2011; 138:85-88

[20] Bharat Bhushan. Biomimetics: Bioinspired Hierarchical-Structured Surfaces for Green Science and Technology. 3rd ed. Switzerland: Springer Nature; 2018.

[21] Raza MA, Kooij ES. Toward Superhydrophobic Surfaces: ControllingWetting Characteristics. Surface and Interface Science: Liquid and Biological Interfaces, $1^{\text {st }}$ ed. Germany: Wiley-VCH Verlag GmbH \& Co. KGaA; 2020. v. 7. p. 391-440

[22] Lee K, Jur JS, Kim DH, Parsons GN. Mechanisms for hydrophilic/hydrophobic wetting transitions on cellulose cotton fibers coated using $\mathrm{Al}_{2} \mathrm{O}_{3}$ atomic layer deposition. J Vac Sci Technol. 2012; A 30:01A163.

[23] Wei DW, Wei H, Gauthier AC, Song J, Jin Y, Huining Xiao H. Superhydrophobic modification of cellulose and cotton textiles: Methodologies and applications. Journal of Bioresources and Bioproducts (JB\&B). 2020; 5:1-15.

[24] Qiao B, Wang TJ, Gao H, Jin Y. High density silanization of nano-silica particles using $\gamma$ aminopropyltriethoxysilane (APTES). Appl Surf Sci. 2015; 351:646-654. 
[25] Xiao Z, Xu J, Yunwei Niu Y, Guangyong Zhu G, Kou X. Effects of Surface Functional Groups on the Adhesion of $\mathrm{SiO}_{2}$ Nanospheres to Bio-Based Materials. Nanomaterials 2019; 9:1411.

[26] Amigoni S, de Givenchy ET, Dufay M, Guittard F. Covalent Layer-by-Layer Assembled Superhydrophobic Organic-Inorganic Hybrid Films. Langmuir. 2009; 25(18):11073-11077.

[27] Cassie, A.; Baxter, S. Wettability of porous surfaces Trans. Faraday Soc. 1944;40:546.

[28] Du P, Wen J, Zhang Z, Song D, Ouahsine, Haibao Hu H. Maintenance of air layer and drag reduction on superhydrophobic surface. Ocean Engineering. 2017; 130: 328-335. 